\title{
PARTICIPA, proyecto de investigación social sobre discapacidad
}

\section{PARTICIPA, social research project about disability}

\section{Palabras clave}

Participación, barreras, facilitadores, discapacidad, derechos.

\section{Keywords}

Participation, barriers, facilitators, disability, rights.

\author{
Blanca Cegarra Dueñas \\ <bcegarra@guttmann.com> \\ Fundació Institut Guttmann. España

\section{Joan Saurí Ruiz} \\ <jsauri@guttmann.com> \\ Fundació Institut Guttmann. España
}

\section{Introducción}

El Institut Guttmann impulsa y dirige el proyecto de investigación e innovación social sobre discapacidad PARTICIPA, una iniciativa compartida con las entidades representadas en el Consejo Social y de Participación de la institución.

PARTICIPA nace con la finalidad de generar conocimiento útil para el colectivo de personas con discapacidad y las entidades sociales, marcándose por objetivo final la transformación social y la mejora de la calidad de vida de las personas con discapacidad. El estudio va dirigido a conocer cómo promocionar la participación en la sociedad de las personas con discapacidad en nuestro país.

\section{Motivación del estudio}

Se espera que la incidencia de personas con discapacidad siga aumentando a medida que la población envejezca, del mismo modo que, gracias a los avances científico-técnicos, se espera que vaya incrementando la

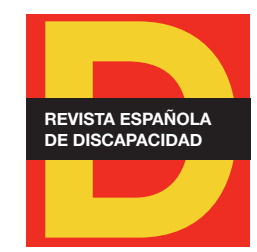

Para citar:

Cegarra, B. y Saurí, J. (2021).

PARTICIPA, proyecto de investigación social sobre discapacidad. Revista Española de Discapacidad, 9(2), pp. 193-203.

Doi: $<$ https://doi.org/10.5569/23405104.09.02.11> 
prevalencia de las personas afectadas por lesiones y/o enfermedades de orígenes diversos. Con la constatación de que la discapacidad no es solo un problema médico, durante los últimos años se ha hecho un esfuerzo considerable para tratar de comprender y medir el impacto social de la misma.

La creciente esperanza de vida tras la adquisición de una discapacidad ha señalado la plena participación en la sociedad como uno de los objetivos finales de un proceso de rehabilitación integral. Esto ha surgido no solo desde un punto de vista biomédico sino también desde una perspectiva social. En las últimas décadas se han realizado esfuerzos significativos para conceptualizar y documentar mejor los resultados de la rehabilitación y de la calidad de la participación en la sociedad de las personas con discapacidad.

La literatura nacional e internacional ha evidenciado que las personas con discapacidad experimentan restricciones sistemáticas en la participación por razón de discapacidad (e.g. Díaz Velázquez, 2017; Kissow, 2015; Friso y Caldin, 2014; Törnbom et al., 2013; Vedeler y Mossige, 2010; Subirats, 2005). Esta situación de discriminación y desigualdad supone una vulneración de derechos para el colectivo con discapacidad y genera exclusión social. En este contexto, consideramos relevante estudiar los factores que impiden la plena participación en la sociedad de las personas con discapacidad, así como los factores facilitadores, con el objetivo de generar conocimiento útil para diseñar estrategias de respuesta innovadoras.

Por otra parte, existe una escasez de datos cuantitativos que ilustren la realidad social de las personas con discapacidad en España de forma global y longitudinal, y que sean de utilidad para la elaboración de políticas públicas (Gosálbez Raull, 2013). Se detecta así la necesidad de poder evidenciar científicamente las restricciones en la participación y las situaciones de discriminación sistemáticas por razón de discapacidad a través de datos objetivos, así como identificar factores facilitadores y estrategias que promocionen la participación de estas personas, y entender cómo se interrelacionan todos estos factores y en qué medida tienen impacto en la participación.

Además, el proyecto que aquí se presenta se encuentra alineado con la Convención sobre los Derechos de las Personas con Discapacidad (ONU, 2006), la cual identifica la necesidad de generar datos y estadísticas sobre la realidad de las personas con discapacidad (artículo 31).

\section{Marco teórico: modelo bio-psico-social}

El marco teórico del estudio se desarrolla a partir de la Clasificación Internacional del Funcionamiento, de la Discapacidad y de la Salud (CIF) publicada por la OMS en el año 2001. Partiendo del modelo bio-psicosocial de la CIF (2001), se estudiará qué factores contextuales (ambientales y personales) influyen en la participación y de qué manera lo hacen, contribuyendo a desarrollar los constructos sociales de la CIF -que actualmente están poco explicados- desde la concepción social de la discapacidad. 
Figura 1. Interacciones entre los componentes de la CIF

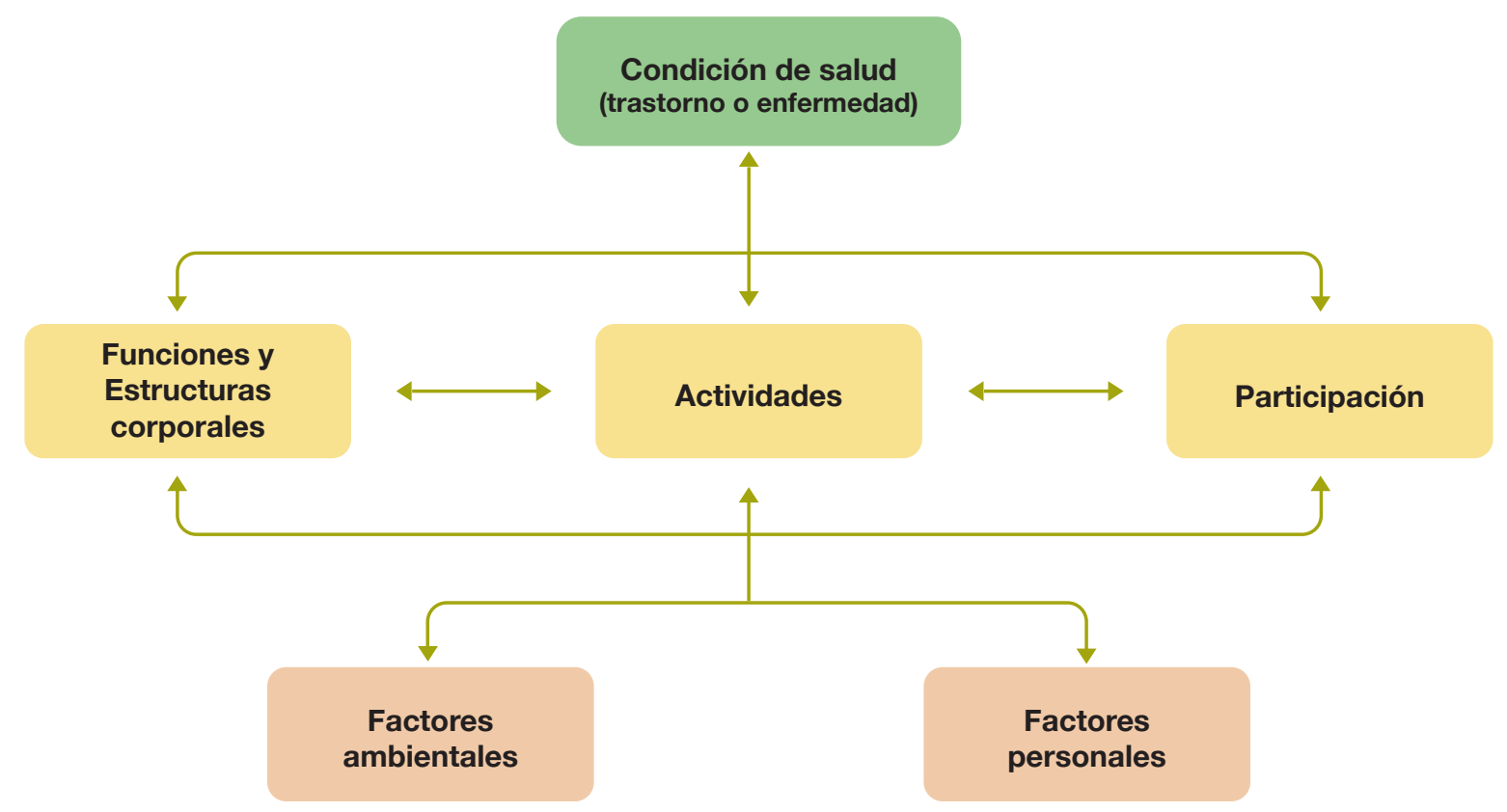

Fuente: Clasificación Internacional del Funcionamiento de la Discapacidad y de la Salud, CIF (OMS, 2001).

La CIF es un lenguaje unificado y estandarizado, a la vez que un marco conceptual que permite analizar cuantitativamente la salud y los estados relacionados con la salud, y comparar datos a nivel internacional (OMS, 2001). Sin embargo, la CIF presenta algunas limitaciones para la investigación social al ser un modelo más médico que social (a pesar de que se define como un modelo integrador, o bio-psico-social). Por lo tanto, se considera necesario para este estudio profundizar en las dimensiones sociales que plantea la CIF y desarrollar y/o revisar algunos de sus dominios, contribuyendo así a convertirla en un marco conceptual más cercano a las concepciones sociales de la discapacidad.

Además, la CIF proporciona constructos y dominios con los que crear un modelo propio que explique el proceso de interacción entre todos los factores de análisis. Como señala la OMS (2001, p. 20), la CIF "proporciona las 'piezas de construcción' para poder crear modelos y estudiar los diferentes aspectos del proceso. En este sentido, podemos considerar la CIF como un idioma y los textos que se pueden crear dependerán de los usuarios, de su creatividad y de su orientación científica". Para esta investigación, nos proponemos explicar las barreras y facilitadores a la participación de las personas con discapacidad haciendo uso del lenguaje propuesto por la CIF, pero superando sus limitaciones anteriormente citadas y contribuyendo a desarrollar los factores contextuales desde una concepción social de la discapacidad. 


\section{Hacia una concepción social de la discapacidad}

En los años 60 el activista estadounidense Ed Roberts, un alumno con discapacidad, ingresa por primera vez en la Universidad de California (Berkeley) después de denunciar las múltiples barreras físicas y sociales que le imponía su entorno y en 1972 inaugura el primer centro de vida independiente junto a otros/as activistas como Judit E. Neumann. Se sitúa en este momento el nacimiento del Movimiento de Vida Independiente, el cual reivindica la autodeterminación de las personas con discapacidad y el derecho a una vida independiente. En otras palabras, la filosofía de vida independiente reivindica el control que la persona debe tener sobre su propia vida y el derecho a ejercer ese control sobre la toma de decisiones que afectan a su proyecto de vida. Fueron muchos los activistas que se sumaron al movimiento social bajo el lema "¡Nada sobre nosotros, sin nosotros!".

Desde entonces, la filosofía de vida independiente se ha ido extendiendo a otros países y su llegada a España se sitúa en el año 2001 de la mano del Foro de Vida Independiente (FVI). A pesar de que cada contexto particular ha hecho suya esta nueva manera de entender la discapacidad, según Frances Hasler (2003), fundadora del Centro Nacional para la Vida Independiente del Reino Unido, este enfoque propone que toda persona: (a) tiene igual valor; (b) tiene la capacidad y tiene que tener la posibilidad de tomar decisiones; (c) tiene el derecho de ejercer control sobre su vida; y (d) tiene el derecho a participar en todas las actividades políticas, económicas y culturales de su comunidad. Así pues, la filosofía de vida independiente pone el foco en el empoderamiento de las personas con discapacidad como medio para que puedan autodeterminar su propio proyecto de vida (en lugar de vivir lo que otros han decidido por ellas), así como en su participación en la sociedad con las mismas condiciones que las demás.

Inspirados por este cambio de paradigma surgido en los Estados Unidos, en el Reino Unido en 1975 los hombres y mujeres con discapacidad reunidos en la Union of Physically Impaired Against Segregation (UPIAS), establecieron los "principios fundamentales de la discapacidad" y elaboraron un manifiesto en el que consideraban que la discapacidad es una situación causada por condiciones sociales, las cuales deben ser eliminadas, y que la sociedad discapacita a las personas y las aísla y excluye de la participación plena en la sociedad por su deficiencia o diferencia funcional.

Más tarde, estas ideas se materializan en el "Modelo social de la discapacidad" descrito por el activista y académico Mike Oliver en la década de los 80, el cual se apuntala en la filosofía de vida independiente, incorporando los principios fundamentales de la UPIAS (1975) que explican la discapacidad como una forma específica de opresión social (Palacios y Romañach, 2006). A partir de aquí, se han desarrollado distintas concepciones sociopolíticas de la discapacidad con diferentes matices. Sin embargo, todos los modelos y concepciones sociales comparten una mirada crítica a la visión individualista, propia de modelos anteriores que se enmarcan en los paradigmas de la prescindencia y de la rehabilitación. Mientras los modelos individualistas centran el problema de la discapacidad en el individuo, los modelos sociales sitúan el foco de la problemática en el entorno y en las barreras que este presenta, ya sean físicas o sociales. Desde esta mirada social, este estudio quiere comprender mejor qué factores facilitan y/o dificultan la participación de las personas con discapacidad y encontrar estrategias de respuesta para las restricciones en la participación.

Las nuevas concepciones sociales han conseguido hacerse un lugar en la ley, materializándose en la Convención sobre los derechos de las personas con discapacidad de las Naciones Unidas (2006), la cual forma 
parte del ordenamiento jurídico español desde el año 2008. La Convención es un instrumento internacional de derechos humanos destinado a proteger los derechos y la dignidad de las personas con discapacidad. Con esta nueva perspectiva, nos dirigimos a un modelo basado en derechos, desde el cual es necesario reclamar la participación plena y efectiva en la sociedad de todas las personas, en igualdad de condiciones que las demás (tal y como establece la Convención).

\section{Dimensiones de estudio}

Partiendo del marco conceptual de la CIF y de la revisión y análisis de literatura sobre la participación de las personas con discapacidad y su interacción con los factores ambientales, se ha empezado la investigación estudiando las siguientes dimensiones (ya que se identifican como aquellas en las que se presentan un mayor número de restricciones en la participación):

Figura 2. Dimensiones de estudio

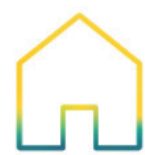

Vivienda

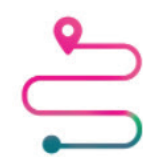

Movilidad

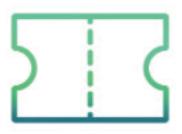

Ocio

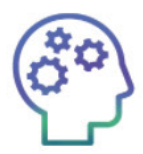

Ocupación

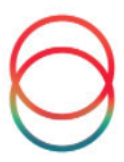

Relaciones interpersonales

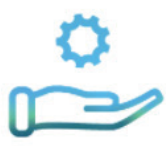

Acceso a servicios

Fuente: elaboración propia.

Para establecer las dimensiones de estudio, se han elaborado definiciones basadas en la Convención que permitan no solo conocer el grado de participación de las personas con discapacidad sino la manera en que participan, para estudiar también la calidad de la participación (esto incluye aspectos como la autodeterminación y la participación en actividades significativas para la persona):

5.1. Vivienda: la vivienda es el lugar que la persona habita. Las personas con discapacidad tienen derecho a escoger su lugar de residencia y dónde y con quién vivir

La Convención de las Naciones Unidas establece que hay que garantizar la accesibilidad de todas las viviendas (art.

9) y el derecho a vivir de forma independiente y ser incluido en la comunidad (art. 19). 
5.2. Movilidad: la movilidad es el conjunto de desplazamientos que realizan las personas para participar en la sociedad. Las personas con discapacidad tienen derecho a desplazarse de la forma y en el momento que lo deseen a un coste asequible

La Convención de las Naciones Unidas establece que hay que adoptar medidas efectivas para asegurar que las personas con discapacidad disfruten de movilidad personal con tanta independencia como sea posible (art. 20).

5.3. Ocupación: por ocupación entendemos el trabajo, el estudio u otra actividad, en la cual la persona dedica el tiempo con retribución o ganancia. Las personas con discapacidad tienen el derecho a acceder a un sistema educativo y a un mercado laboral inclusivos y accesibles

La Convención de las Naciones Unidas reconoce el derecho de las personas con discapacidad a trabajar, en igualdad de condiciones que las otras; esto incluye el derecho a tener la oportunidad de ganarse la vida mediante un trabajo libremente elegido o aceptado en un mercado y un entorno laborales que sean abiertos, inclusivos y accesibles a las personas con discapacidad (art. 27). Además, la Convención insta a los Estados Parte a asegurar que las personas con discapacidad tengan acceso general a la educación superior, la formación profesional, la educación para adultos y el aprendizaje durante toda la vida sin discriminación y en igualdad de condiciones que las demás (art. 24).

5.4. Ocio: entendemos por ocio el acceso a las actividades con fines culturales, lúdicas, recreativas y deportivas. Las personas con discapacidad tienen derecho a acceder a espacios (físicos o digitales) de oferta cultural, lúdica, recreativa o deportiva diseñados para todas las personas (espacios accesibles e inclusivos)

La Convención de las Naciones Unidas reconoce el derecho de las personas con discapacidad a participar, en igualdad de condiciones que las otras, en la vida cultural, las actividades recreativas, el esparcimiento y el deporte (art. 30).

5.5. Relaciones interpersonales: la relación interpersonal es la interacción de un individuo con las personas de su entorno

La Convención de las Naciones Unidas insta a los Estados a tomar medidas efectivas y pertinentes para poner fin a la discriminación contra las personas con discapacidad en todas las cuestiones relacionadas con el matrimonio, la familia, la paternidad y las relaciones personales (art. 23).

5.6. Servicios: el acceso a servicios (públicos y de uso público) accesibles e inclusivos, como los servicios de salud, sociales, de educación, restauración, comercios, etc. Las personas con discapacidad deben tener la oportunidad de acceder a los servicios en igualdad de condiciones que las otras

La Convención de las Naciones Unidas establece que, a fin de que las personas con discapacidad puedan vivir independientemente y participar plenamente en todos los aspectos de la vida, hay que adoptar medidas pertinentes 
para asegurar el acceso de todas las personas al entorno físico, el transporte, la información y las comunicaciones, incluidos los sistemas y las tecnologías de la información y las comunicaciones, y a otros servicios e instalaciones abiertos al público o de uso público, tanto en zonas urbanas como en rurales (art. 9).

Para cada una de estas dimensiones, se estudiará el grado y la calidad de la participación de las personas con discapacidad, así como las barreras y facilitadores que determinan esta participación.

Las barreras pueden ser de carácter físico o social. Las barreras físicas a menudo son fácilmente identificables, por ejemplo, la presencia de unas escaleras para entrar en un restaurante o en un centro comercial. De acuerdo con la Convención de las Naciones Unidas, ratificada por España en 2008, la presencia de estas barreras supone una vulneración de derechos.

Las barreras sociales, en cambio, no son tan evidentes. Esto hace que se den situaciones de discriminación diariamente y no seamos conscientes de ello. Por ejemplo, cuando una persona con discapacidad se vuelve invisible cuando va acompañada de una persona que no tiene discapacidad, cuando entra en un restaurante y preguntan a su pareja: “¿Dónde se sentará? ¿Dónde la colocaremos?”, como si aquella persona no estuviera presente. Varios investigadores sociales señalan que, para poder identificar el origen de todas estas barreras, nos tenemos que referir a otro concepto, el capacitismo, el cual está muy arraigado en el imaginario social.

El capacitismo (ableism) es el conjunto de creencias y prácticas cotidianas que señalan la idea que hay un determinado tipo de cuerpo y mente que es el estándar de la especie humana, el estándar con el cual todo el mundo se tiene que comparar, aquel tipo de sujeto y de cuerpo que se proyecta normativamente como lo perfecto y típico de la especie y, por lo tanto, como lo que es esencial y plenamente humano (Campbell, 2008).

El capacitismo está implícitamente insertado en la forma de construir entornos y regula las expectativas sobre cómo son las personas que los habitan. En cuanto las personas con discapacidad se alejan de este estándar, se encuentran con barreras que dificultan su participación en la sociedad. En la medida en que transformemos los entornos en espacios accesibles e inclusivos podremos garantizar la participación de todas las personas en igualdad de condiciones.

\section{Objetivos de estudio}

El proyecto PARTICIPA se centra en el análisis de la participación en la sociedad de las personas con discapacidad para dar respuesta a la siguiente pregunta de investigación:

¿Cuáles son los factores facilitadores y las barreras a la participación en la sociedad de las personas con discapacidad?

Así, el objeto de estudio se centra en la interacción entre la participación y los factores ambientales. Una mejor comprensión de los factores determinantes de la participación permitirá diseñar estrategias innovadoras que promocionen la participación plena y efectiva de las personas con discapacidad. 
Los objetivos específicos del proyecto son:

- Evaluar, codificar y calificar la participación de las personas con discapacidad e identificar factores determinantes de la participación (principalmente barreras y facilitadores).

- Analizar la influencia de los factores determinantes en la participación de las personas con discapacidad.

- Comprender las relaciones entre los factores que influyen en la participación que ayuden a orientar las intervenciones y recursos destinados a reducir las restricciones y promocionar los factores facilitadores de la participación.

Para lograr estos objetivos se está llevando a cabo un estudio longitudinal prospectivo de cohortes en el que se espera que participen un mínimo de 2000 personas con discapacidad voluntarias a través de una web, una herramienta innovadora que permite la recogida y el análisis de datos sobre las barreras y los facilitadores de la participación con los que conviven las personas con discapacidad. Los datos recogidos de las personas con discapacidad mayores de 18 años y residentes en España o Andorra se estratificarán por edad, género, tipo de discapacidad, situación socioeconómica y procedencia; lo que permitirá realizar un análisis interseccional del objeto de estudio.

Además, también pueden participar en el estudio personas de apoyo (asistente personal, traductor vital, familiar, amigo...) que se registren y que acompañen a la persona con discapacidad en su participación o participen en nombre de la persona con discapacidad.

\section{- Web del proyecto}

El estudio se vehicula a través de una web que ya está activa y en funcionamiento (https://participa.guttmann.com/es/), la cual recoge la información a través de una serie de cuestionarios online y un foro de participación (https://participa.guttmann.com/foro/). La recogida de datos se realiza a través de esta web, donde cada persona participante puede responder a una serie de cuestionarios en "su espacio personal", accediendo a través de un nombre de usuario y una contraseña únicos e intransferibles.

Por otra parte, la web ofrece espacios de participación directa a través de un foro online organizado en 6 subforos, uno por cada una de las 6 dimensiones: vivienda, movilidad, ocupación, ocio, relaciones interpersonales y servicios. Además, las temáticas del foro se podrán ir ampliando en función de la demanda y el interés de los participantes y crear así nuevos subforos. En cada uno de estos subforos, las personas participantes en el proyecto podrán aportar reflexiones, opiniones, comentarios, compartir experiencias sobre situaciones específicas, plantear problemas y proponer soluciones nacidas de circunstancias vividas en situaciones similares. Cada uno de los subforos estará moderado por especialistas en cada una de las dimensiones.

La participación en el foro permite de este modo denunciar restricciones en la participación a la vez que aportar y recibir opiniones, soluciones o estrategias innovadoras. Es una plataforma de recogida de información cualitativa que será de gran valor para los objetivos de estudio y, a la vez, funciona como un espacio de apoyo entre iguales en el que compartir problemas y soluciones. Cada mensaje en el foro puede ser acompañado de imágenes o vídeos para ilustrar la situación. 


\section{Impacto esperado}

El proyecto PARTICIPA pretende tener un impacto social positivo para la sociedad en general y para el colectivo de personas con discapacidad en particular. El conocimiento social generado a través del proyecto permitirá profundizar en el análisis de la participación de las personas con discapacidad y, por lo tanto, diseñar estrategias de respuesta basadas en la evidencia científica y surgidas directamente del colectivo (lógica bottom-up), huyendo así de la mirada paternalista. Este conocimiento no solo tiene valor desde el punto de vista científico sino que es información de utilidad para los grupos de interés o stakeholders del ámbito de la discapacidad (asociaciones de personas con discapacidad, otras entidades sociales, decisores políticos, profesionales, etc.).

Además, este proyecto da visibilidad a las situaciones que vulneran el derecho de participación de las personas con discapacidad, haciendo pedagogía acerca de las discriminaciones existentes con la finalidad de promocionar la conciencia social y el cambio de mirada hacia la discapacidad.

\section{Figura 3. Impacto del proyecto PARTICIPA}

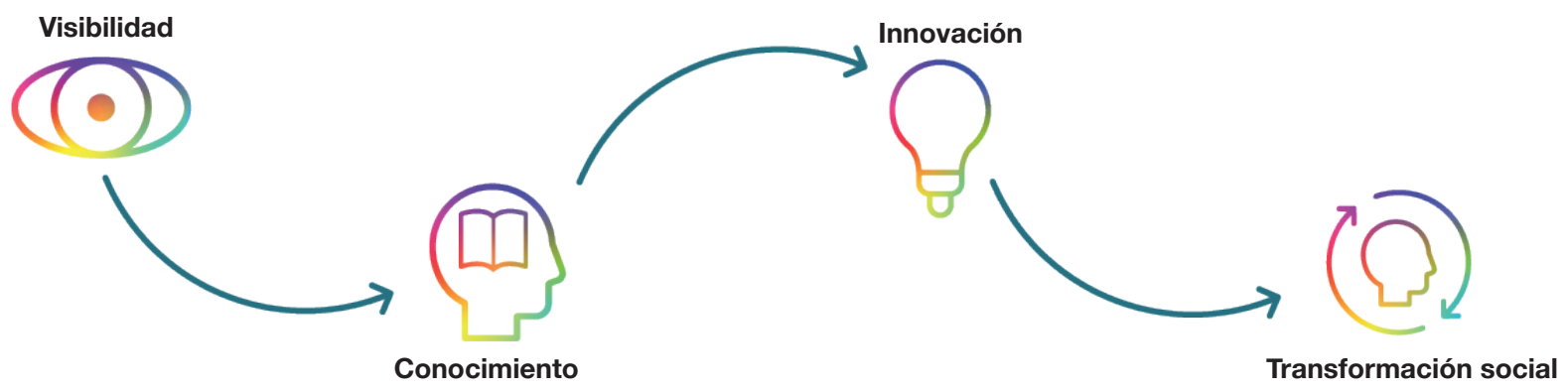

Fuente: elaboración propia.

En resumen, PARTICIPA es un proyecto innovador para transformar nuestra sociedad a partir de:

- Visibilizar: hacer visibles las restricciones y barreras existentes por razón de discapacidad. Aumentar la conciencia social hacia las situaciones de vulneración de derechos que viven las personas con discapacidad. Hacer pedagogía para fomentar un cambio de mirada hacia la discapacidad, y transitar del modelo médico al modelo social. PARTICIPA será un altavoz de las necesidades expresadas por el colectivo, dando voz a las personas con discapacidad sobre cómo quieren participar en la sociedad.

- Generar conocimiento: generación de datos, conocimiento y evidencia que permita diseñar estrategias innovadoras para dar respuesta a las restricciones existentes y suprimir barreras.

- Innovación social: diseñar nuevas propuestas, ideas, estrategias, servicios..., orientados a promocionar la participación a la sociedad de las personas con discapacidad en igualdad de condiciones que el resto. 
- Transformación social: hacer difusión entre todos los agentes sociales implicados sobre nuevos conocimientos, buenas prácticas y estrategias innovadoras que transformen la realidad, con el objetivo de conseguir su aplicación para generar cambios en la cultura y el funcionamiento de nuestra sociedad.

PARTICIPA es un proyecto de investigación social dirigido a conocer y entender cómo se puede promocionar la participación en la sociedad de las personas con discapacidad, con la voluntad de transformarla para que sea plenamente inclusiva.

Las personas que participen en el estudio conocerán qué les puede ayudar a incrementar la participación y recibirán recomendaciones para hacerlo en una efectiva igualdad de oportunidades. jJuntos suprimimos barreras y construimos una sociedad mejor!

Si eres mayor de edad y tienes una discapacidad, entra en la web y iparticipa!

https://participa.guttmann.com/es/ 


\section{Referencias bibliográficas}

Campbell, F. K. (2008). Refusing able(ness): A preliminary conversation about ableism. M/C Journal, $11(3), 9$ p. https://doi.org/10.5204/mcj.46.

Díaz Velázquez, E. (2017). El acceso a la condición de ciudadanía de las personas con discapacidad en España. Un estudio sobre la desigualdad por razón de discapacidad. Comité Español de Representantes de Personas con Discapacidad; Ediciones Cinca.

Friso, V. y Caldin, R. (2014). Capability, work and social inclusion. Procedia-Social and Behavioral Sciences, 116, pp. 4914-4918. https://doi.org/10.1016/j.sbspro.2014.01.1049.

Gosálbez Raull, M. B. (2013). Estadísticas de discapacidad: estado de situación, necesidad y perspectivas. Economía española y Protección Social, (5), pp. 169-202.

Hasler, F. (2003). El concepto de vida Independiente a través de tres visiones: filosófica, socio-política y económica. En J. V. García (ed.), El movimiento de vida independiente: experiencias internacionales (pp. 55-76). Fundación Luis Vives.

Kissow, A. M. (2015). Participation in physical activity and the everyday life of people with physical disabilities: A review of the literature. Scandinavian Journal of disability research, 17(2), pp. 144-166. http://doi.org/10.1080/ 15017419.2013 .787369$.

Organización Mundial de la Salud (2001). Clasificación Internacional del Funcionamiento de la Discapacidad y de la Salud. Ministerio de Trabajo y Asuntos Sociales. https://www.imserso.es/InterPresent2/groups/imserso/ documents/binario/435cif.pdf.

Organización de las Naciones Unidas (2006). Convención sobre los derechos de las personas con discapacidad y protocolo facultativo. ONU. https://www.un.org/disabilities/documents/convention/convoptprot-s.pdf.

Palacios, A. y Romañach, J (2006). El modelo de la diversidad. La Bioética y los derechos humanos como herramientas para alcanzar la plena dignidad en la diversidad funcional. Ediciones Diversitas-AIES.

Subirats, J. (dir.) (2005). Análisis de los factores de exclusión social. Fundación BBVA.

Törnbom, K et al. (2013). Experiences of participation in a Swedish society among adults with cerebral palsy or spina bifida: Involvement and challenges. Journal of Social Work in Disability \& Rehabilitation, 12(4), pp. 256271. https://doi.org/10.1080/1536710X.2013.834783.

UPIAS (1975). Fundamental principles of disability. The Union of Physically Impaired Against Segregation.

Vedeler, J. S. y Mossige, S. (2010). Pathways into the labour market for Norwegians with mobility disabilities. Scandinavian Journal of Disability Research, 12(4), pp. 257-271. https://doi:10.1080/15017410903581189. 